\title{
Is Obstructive Sleep Apnea A Risk Factor for Severe Acute Respiratory Syndrome Coronavirus 2 Infection?
}

\author{
Elif Torun Parmaksız ${ }^{1 *}$ and Ergün Parmaksız ${ }^{2}$ \\ ${ }^{1}$ Health Sciences University, Sancaktepe Şehit Prof Ilhan Varank Training Hospital, Department of Chest diseases - Istanbul, \\ Turkey
}

${ }^{2}$ Health Sciences University, Kartal Dr Lutfi Kirdar City Hospital, Department of Nephrology - Istanbul, Turkey

*Corresponding author: Elif Torun Parmaksı, HSU, Sancaktepe Șehit Prof İlhan Varank Training Hospital, Department of Chest diseases - Istanbul, Turkey

\section{ARTICLE INFO \\ ABSTRACT}

Received: 慧 October 05, 2021

Background: Coronavirus Disease 2019 (COVID-19) and obstructive sleep apnea

Published: 慧 October 18, 2021

Citation: Elif Torun Parmaksız, Ergün Parmaksiz. Is Obstructive Sleep Apnea A Risk Factor for Severe Acute Respiratory Syndrome Coronavirus 2 Infection?. Biomed J Sci \& Tech Res 39(3)-2021. BJSTR. MS.ID.006298.

Keywords: Obstructive Sleep Apnea; COVID-19; SARS-CoV-2; Coronavirus; Apnea-Hypopnea Index (OSA) share many demographic characteristics and comorbidities. We aimed to evaluate the prevalence of severe acute respiratory syndrome coronavirus-2 (SARS-CoV-2) infection in patients diagnosed with OSA and the effect of OSA on the severity of the infection in these patients.

Material and Methods: In this retrospective observational study, the records of cases who had polysomnography (PSG) confirmed OSA in the last five years were reviewed. The electronic medical records were queried for the results of the SARS-CoV-2 polymerase-chain-reaction (PCR) tests in this population. For patients with positive tests, the demographic data, PSG results, clinical, laboratory, and radiological findings of COVID-19 were recorded.

Results: Of 1317 OSA patients, we identified 14 patients with positive PCR results for SARS-CoV-2. All the patients underwent outpatient treatment, and no hospital or intensive care unit (ICU) admission, progression to respiratory failure or mortality was observed.

Conclusion: Patients with COVID-19 who had OSA were not likely to have a severe infection. We demonstrated that OSA cannot be considered as one of the underlying medical conditions predisposing to increased risk or poor outcome in COVID-19.

\section{Introduction}

Severe Acute Respiratory Syndrome Coronavirus 2 (SARSCoV-2) has emerged as public health crises first in the city of Wuhan, Hubei province in China in December 2019, and has subsequently turned out to be a global problem. Coronavirus Disease 2019 (COVID-19) may present in a wide spectrum of clinical forms ranging from mild symptoms such as fever, cough, or fatigue to severe pneumonia, septic shock, organ failure, or death. Understanding risk factors for disease susceptibility and severity is essential to prioritize target populations and patients that are at most risk. Which factors predict the susceptibility to COVID-19 and the severity of the infection have been studied earlier. Diabetes mellitus (DM), hypertension, respiratory and cardiovascular disorders are among the factors with high consistency of association to lifethreatening outcomes [1]. COVID-19 and obstructive sleep apnea (OSA) share many demographic characteristics and comorbidities such as advanced age, male gender, obesity, hypertension, cardiac complications, and DM. Both OSA and COVID 19 are associated with pro-inflammatory mediators. Coronavirus- 2 enters the cell with the help of the Angiotensin-Converting Enzyme-2 (ACE-2) receptor. The number of ACE- 2 receptors in adipose tissue increases in 
obesity [2]. Hypoxemia in OSA may affect the coagulation cascade and enhance the tendency to coagulation caused by COVID 19 [3]. Taken together, sharing the mentioned putative risk factors in common, is OSA risk and poor prognostic factor for COVID-19 infection?

There are several studies focused on the frequency of OSA in COVID-19 patients and the effect of OSA on the prognosis of COVID-19 [4,5]. However, there are not enough data in the literature regarding the prevalence of COVID-19 in the population with OSA In our study, we aimed to evaluate the prevalence of severe acute respiratory syndrome coronavirus-2 (SARS-CoV-2) infection in patients diagnosed with OSA and the effect of OSA on the severity of the infection in these patients.

\section{Material and Methods}

We conducted a retrospective observational study in the OSA population diagnosed by polysomnography (PSG) in our clinic. The records of consecutive patients who underwent PSG between March 2015 and March 2020 in our clinic were reviewed. OSA was diagnosed using overnight PSG. The standard overnight PSG included electroencephalography, electrooculography, submental and bilateral leg electromyography, and electrocardiography recordings. We measured airflow with a nasal pressure transducer and an oronasal thermistor, respiratory effort via respiratory inductance plethysmography, and arterial oxyhemoglobin saturation via a finger pulse oximeter. Experienced technicians collected and digitalized all signals using computerized PSG systems (Comet Grass: Astro-Med, Inc., West Warwick, Rhode Island, United States, and Viasys Cephalo-Pro, SomnoStar: VIASYS Healthcare, Hochberg, Germany) following established standards [6]. Certified sleep specialists, experienced in sleep medicine, scored sleep stages using the American Academy of Sleep Medicine (AASM) scoring system $[7,8]$. Grading of the apnea-hypopnea index (AHI) followed AASM's 1999 criteria as follows: an AHI less than 5 was normal, an AHI higher than 5 but less than 15 was mild, an AHI higher than 15 but less than 30 was moderate and an AHI higher than 30 was severe [9].

The electronic medical records of the Public Health Management System were queried for the results of the SARS-CoV-2 polymerasechain-reaction (PCR) tests for all these subjects with PSG confirmed sleep disorders. Whether the subjects have been tested for SARSCoV-2 with PCR and the test results were recorded. For patients with positive tests, the demographic data, results, and diagnoses of the sleep study were recorded. Comorbidities were ascertained by ICD-10-CM coding and medical record data. A case of Covid-19 was defined by a positive result on a PCR assay of a specimen collected on a nasopharyngeal swab. The clinical findings, laboratory and radiological data, outpatient/inpatient treatment status, and the course of the COVID-19 of the patients with positive PCR tests were recorded.

\section{The Study Protocol was Approved by the Local Ethics Committee}

All statistical analyses were performed using SPSS software (version 17.0). For baseline characteristics, mean (standard deviation) for continuous variables and number and percentages for categorical variables were calculated. Given that this is a descriptive study, no analysis for statistical significance was performed.

\section{Results}

Our analysis included 1317 OSA patients diagnosed by PSG. A review of the medical records demonstrated that 51 patients have been tested for SARS-CoV-2 with PCR. The reasons for testing were suspicion of infection, contact tracing, scanning before hospital admission or interventional procedures, or screening for travel. We identified 14 patients with positive PCR results for SARSCoV-2 (Figure 1). The mean age of the 14 patients was $48.9 \pm 12.1$ years. The majority of the patients were male $(n=13,93 \%)$. The mean BMI was $29.7 \pm 2.4 \mathrm{~kg} / \mathrm{m} 2$. The polysomnographic data is demonstrated in Table 1. Eight (57\%) cases had mild OSA, three $(21 \%)$ had moderate OSA, and three (21\%) had severe OSA. Three cases were asymptomatic. Main complaints were chest pain $(n=6$, $43 \%)$, fever $(n=5,36 \%)$, fatigue $(n=3,21 \%)$, cough $(n=3,21 \%)$, shortness of breath $(n=3,21 \%)$, loss of taste and smell $(n=2$, $14 \%)$, and diarrhea $(n=1,7 \%)$. Two patients $(14 \%)$ had DM and two $(14 \%)$ had hypertension. Two patients $(14 \%)$ did not need radiological evaluation. Others underwent computed tomography (CT) scanning; normal CT findings was observed in six cases (43\%); involvement was unilateral in three cases (21\%) and bilateral in three $(21 \%)$ cases. The mean percentage of oxygen saturation was $97.4 \pm 3.0(90-99)$ on initial evaluation. The laboratory data of COVID-19 patients is demonstrated in Table 2. All the patients underwent outpatient treatment and no hospital or intensive care unit (ICU) admission, progression to respiratory failure or mortality was observed.

Table 1: The polysomnographic data of OSA patients with COVID-19.

\begin{tabular}{|c|c|}
\hline & Mean \pm standard deviation \\
\hline AHI & $25.65 \pm 30.03$ \\
\hline AI & $18.05 \pm 29.41$ \\
\hline Time elapsed $<\mathrm{SpO}_{2} \% 90(\%)$ & $15.01 \pm 23.21$ \\
\hline ODI & $27.33 \pm 28.94$ \\
\hline Minimum $\mathrm{SpO}_{2}$ & $81.61 \pm 8.24$ \\
\hline Mean $\mathrm{SpO}_{2}$ & $92.01 \pm 4.31$ \\
\hline
\end{tabular}

saturation

AHI: apnea-hypopnea index (events per hour); AI: apnea index (events per hour); ODI: oxygen desaturation index (events per hour); spO2: Arterial oxygen three( $21 \%$ ) had moderate OSA, and three $(21 \%)$ had severe OSA. 
Table 2: The laboratory findings of COVID-19 cases.

\begin{tabular}{|c|c|c|}
\hline & Mean \pm standard deviation & Minimum-maximum \\
\hline C-reactive protein $(\mathrm{mg} / \mathrm{L})$ & $24.74 \pm 31,68$ & $2.98-83.40$ \\
\hline Total lymphocyte count & $1741 \pm 894$ & $150-2310$ \\
\hline D-dimer $(\mu \mathrm{g} / \mathrm{L})$ & $767 \pm 1032$ & $58.20-203.00$ \\
\hline Ferritin $(\mu \mathrm{g} / \mathrm{L})$ & $130.60 \pm 102.38$ & $90-99$ \\
\hline sp02 $(\%)$ & $97.42 \pm 3.00$ & \\
\hline
\end{tabular}

$\mathrm{spO}_{2}$, Arterial oxygen saturation

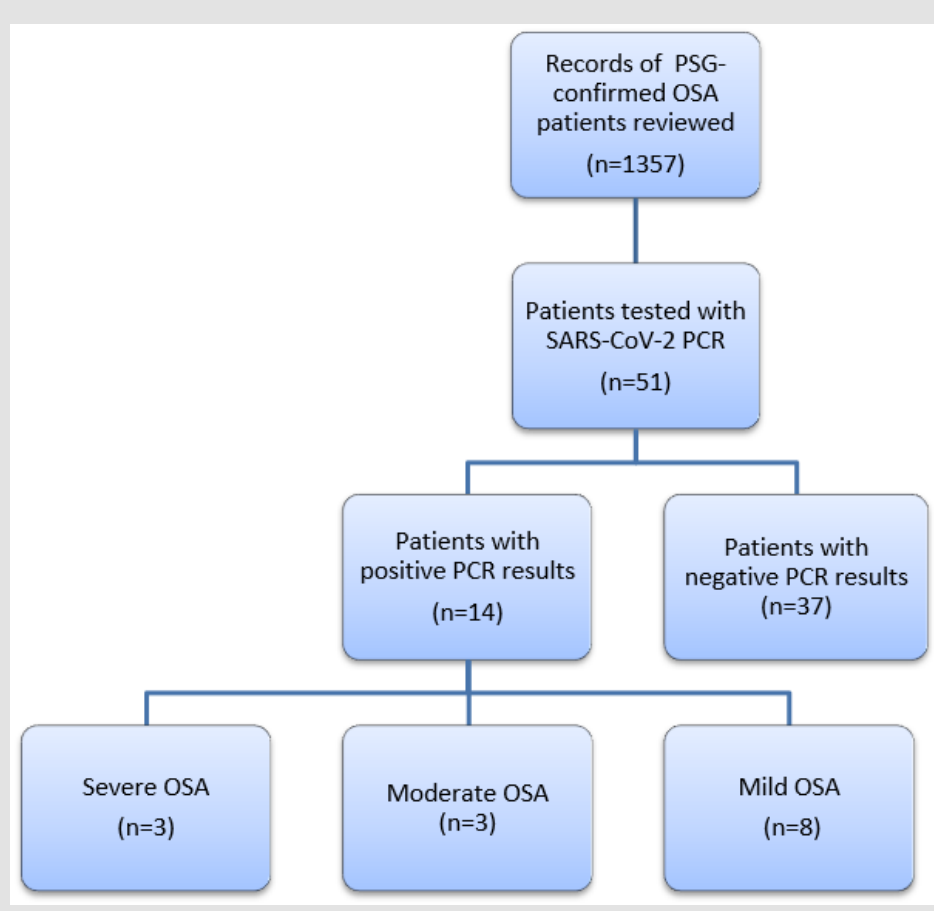

Figure 1.

\section{Discussion}

We have observed that the prevalence of COVID-19, the need for hospitalization, and progression to respiratory failure, namely severe infection did not seem to increase in OSA patients. In our large OSA population, no hospital admission or death occurred due to COVID-19. Recognition of conditions substantially associated with significant morbidity and mortality is essential to offer prudent preventive measures to vulnerable populations. Theoretically, OSA patients should have increased susceptibility and severity for SARS-CoV-2 infection as they share essentially identical risk factors. Due to overlapping predisposing factors, OSA patients are thought to show a heightened risk of poor outcomes in the case of COVID-19. Our findings are contradictory to this fact. Several studies including a small population of severe COVID-19 patients have shown that $21-28 \%$ of patients had OSA $(10,11) \mathrm{A}$ recent study on the relationship between OSA and risk of COVID-19 infection has revealed that the risk for COVID-19 infection was about 8-fold greater in OSA patients. The authors stated that the risk of hospitalization and respiratory failure increased, as well [12]. Obesity predisposes to OSA [13]. Links between obesity and COVID-19 have been investigated. In a recent analysis, obesity has been reported as an independent risk factor for invasive mechanical ventilation in COVID-19 patients [14].

Up through January 22, 2021, a total of 28.195.901 tests have been applied and 2.418.472 people had tested positive for the new SARS-CoV-2 coronavirus in Turkey. The total number of deaths is 24789 [15]. These data reveal that about $3 \%$ of the Turkish population has been infected with SARS-CoV-2. Concerning these data, the prevalence of COVID-19 in our selected population does not seem to be higher than the general population. One possible explanation is the fact that this specific cohort, as well as their families are aware of the increased risk of morbidity and mortality from Covid-19, due to their comorbid medical conditions, such as obesity, hypertension, diabetes, and thus, they were extra cautious 
about exposures. One major problem with treatment in OSA is nonadherence to CPAP treatment. CPAP adherence has been shown to improve significantly during the COVID-19 lockdown [16]. Staying at home, travel restrictions, and the fear of having a poor prognosis, and the probability of being hospitalized might have been motivating factors in better CPAP adherence [16]. This may be considered as a protective factor for OSA patients. The current study has several limitations. Coding and recording of data may be imprecise and missing. Still, the administrative data we based our investigation on is very reliable. Our data reflect OSA as it is diagnosed by PSG. However, OSA is widely underdiagnosed, therefore the true prevalence is probably higher. It may be argued that some OSA patients might have PCR negative COVID-19 infection, on the other hand, the same argument may be true for all the population. In the population we reviewed, all OSA patients who had negative PCR results have been on basis of screening. They had no infection symptoms or signs.

In contrary to previous reports suggesting an increased risk of COVID-19 in OSA patients, our study represents novel data on the incidence of COVID-19 in population with confirmed OSA. To our knowledge, this is the first study to claim that susceptibility, severity, and mortality are not increased in COVID-19 patients with sleep disorders.

\section{Conclusion}

Our results provide some initial data regarding COVID-19 risk in a large OSA population. We demonstrated that OSA cannot be considered as one of the underlying medical conditions predisposing to increased risk or poor outcome in COVID-19. Poor COVID-19 related prognosis, if exists, may be attributed to other risk factors or comorbidities accompanying OSA. We have observed that the prevalence of COVID-19, the need for hospitalization, and progression to respiratory failure, namely severe infection did not seem to increase in OSA patients. In our large OSA population, no hospital admission or death occurred due to COVID-19. Recognition of conditions substantially associated with significant morbidity and mortality is essential to offer prudent preventive measures to vulnerable populations. Theoretically, OSA patients should have increased susceptibility and severity for SARS-CoV-2 infection as they share essentially identical risk factors. Due to overlapping predisposing factors, OSA patients are thought to show a heightened risk of poor outcomes in the case of COVID-19. Our findings are contradictory to this fact. Several studies including a small population of severe COVID-19 patients have shown that 21$28 \%$ of patients had OSA $[10,11]$ A recent study on the relationship between OSA and risk of COVID-19 infection has revealed that the risk for COVID-19 infection was about 8-fold greater in OSA patients.
The authors stated that the risk of hospitalization and respiratory failure increased, as well [12]. Obesity predisposes to OSA [13]. Links between obesity and COVID-19 have been investigated. In a recent analysis, obesity has been reported as an independent risk factor for invasive mechanical ventilation in COVID-19 patients [14]. Up through January 22, 2021, a total of 28.195.901 tests have been applied and 2.418.472 people had tested positive for the new SARS-CoV-2 coronavirus in Turkey. The total number of deaths is 24789 [15]. These data reveal that about $3 \%$ of the Turkish population has been infected with SARS-CoV-2. Concerning these data, the prevalence of COVID-19 in our selected population does not seem to be higher than the general population. One major problem with treatment in OSA is nonadherence to CPAP treatment. CPAP adherence has been shown to improve significantly during the COVID-19 lockdown [16]. Staying at home, travel restrictions, and the fear of having a poor prognosis, and the probability of being hospitalized might have been motivating factors in better CPAP adherence [16]. This may be considered as a protective factor for OSA patients. The current study has several limitations. The study does not include a control group to determine the prevalence of hospitalization or severe disease in a cohort without OSA. Still, we have the prevalances from the total population to compare the prevalence of the cohort. Coding and recording of data may be imprecise and missing. Still, the administrative data we based our investigation on is very reliable. Our data reflect OSA as it is diagnosed by PSG. However, OSA is widely underdiagnosed, therefore the true prevalence is probably higher. It may be argued that some OSA patients might have PCR negative COVID-19 infection, on the other hand, the same argument may be true for all the population. In the population we reviewed, all OSA patients who had negative PCR results have been on basis of screening. They had no infection symptoms or signs.

In contrary to previous reports suggesting an increased risk of COVID-19 in OSA patients, our study represents novel data on the incidence of COVID-19 in population with confirmed OSA. To our knowledge, this is the first study to claim that susceptibility, severity, and mortality are not increased in COVID-19 patients with sleep disorders. In conclusion, our results provide some initial data regarding COVID-19 risk in a large OSA population. We demonstrated that OSA cannot be considered as one of the underlying medical conditions predisposing to increased risk or poor outcome in COVID-19. Poor COVID-19 related prognosis, if exists, may be attributed to other risk factors or comorbidities accompanying OSA.

\section{Compliance with Ethical Standards}

No Funding was received.

Elif Torun Parmaksız declares that she has no conflict of interest. 
Ergün Parmaksiz declares that he has no conflict of interest.

Ethical approval: All procedures performed in studies involving human participants were in accordance with the ethical standards of the institutional and/or national research committee and with the 1964 Helsinki declaration and its later amendments or comparable ethical standards. The study protocol was approved by the local ethics committee (Kartal Dr. Lutfi Kirdar Ethics Committee Approval number:314/194/44)

This type of study does not require informed consent.

\section{References}

1. Ejaz H, Alsrhani A, Zafar A, Javed H, Junaid K, et al. (2020) COVID-19 and comorbidities: Deleterious impact on infected patients. J Infect Public Health. 13(12): 1833-1839.

2. Iannelli A, Favre G, Frey S, Vincent Esnault, Jean Gugenheim, et al. (2020) Obesity and COVID 19: ACE 2, the missing tile. Obes Surg 30(11): 46154617.

3. Suquia AG, Alonso-Fernández A, De La Peña M, David Romero, Javier Piérola, et al. (2009) High D-dimer levels after stopping anticoagulants in pulmonary embolism with sleep apnoea. Eur Respir J 46(6): 16911700 .

4. Feuth T, Saaresranta T, Karlsson A, Valtonen M, Peltola V, et al. (2020) Is sleep apnea a risk factor for COVID-19? Findings from a retrospective cohort study. Sleep Med Dis Int J. Published online October 22, 2020.

5. Miller MA, Cappuccio FP (2020) A systematic review of COVID-19 and obstructive sleep apnoea, Sleep Medicine Reviews, volume 55, 2021 101382, ISSN 1087-0792.

6. Kushida CA, Littner MR, Morgenthaler M, Cathy A Alessi, Dennis Bailey, et al. (2005) Practice parameters for the indications for polysomnography and related procedures: an update for 2005. Sleep 284(4): 499-521.

\section{ISSN: 2574-1241}

DOI: $10.26717 /$ BJSTR.2021.39.006298

Elif Torun Parmaksız. Biomed J Sci \& Tech Res

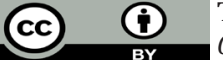

This work is licensed under Creative Commons Attribution 4.0 License

Submission Link: https://biomedres.us/submit-manuscript.php
7. Berry RB, Brooks R, Gamaldo C, Harding SM, Lloyd RM, et al. (2017) AASM Scoring Manual Updates for 2017 (Version 2.4). J Clin Sleep Med 13(5): 665-666.

8. Michael J Sateia (2014) American Academy of Sleep Medicine. International Classification of Sleep Disorders, $3^{\text {rd }}$ edDarien, IL: American Academy of Sleep Medicine; 2014. 146(5): 1387-1394.

9. Carl Stepnowsky, Tania Zamora, Christine Edwards (1999) Sleeprelated breathing disorders in adults: recommendations for syndrome definition and measurement techniques in clinical research. The Report of an American Academy of Sleep Medicine Task Force. Sleep; 1999; 225: 667-689.

10. Bhatraju PK, Ghassemieh BJ, Nichols M, Richard Kim, Keith R Jerome, et al. (2020) Covid-19 in critically ill patients in the Seattle region-case series. N Engl J Med. Published online March 30, 2020.

11. Arentz M, Yim E, Klaff L, Sharukh Lokhandwala, Francis X Riedo, et al. (2020) Characteristics and outcomes of 21 critically ill patients with COVID-19 in Washington State. JAMA 323(16): 1612-1614.

12. Maas MB, Kim M, Malkani RG, Abbott SM, Zee PC, et al. (2020) Obstructive Sleep Apnea and Risk of COVID-19 Infection, Hospitalization and Respiratory Failure. Sleep Breath. 2020 Sep 29: 1-3.

13. Romero-Corral A, Caples SM, Lopez-Jimenez F, Somers VK (2010) Interactions between obesity and obstructive sleep apnea: implications for treatment. Chest 137(3): 711-719.

14. Simonnet A, Chetboun M, Poissy J, Violeta Raverdy, Jerome Noulette, et al. (2020) High prevalence of obesity in severe acute respiratory syndrome coronavirus-2 (SARS-CoV-2) requiring invasive mechanical ventilation. Obesity (Silver Spring). Published online April 9, 2020. 28(7): 1195-1199.

15. (2020) Turkey COVID-19 Patient Table, General Coronovirus Table. Republic of Turkey, Ministry of Health.

16. Attias D, Pepin JL, Pathak A (2020) Impact of COVID-19 lockdown on adherence to continuous positive airway pressure by obstructive sleep apnoea patients. Eur Respir J 56(1): 2001607.

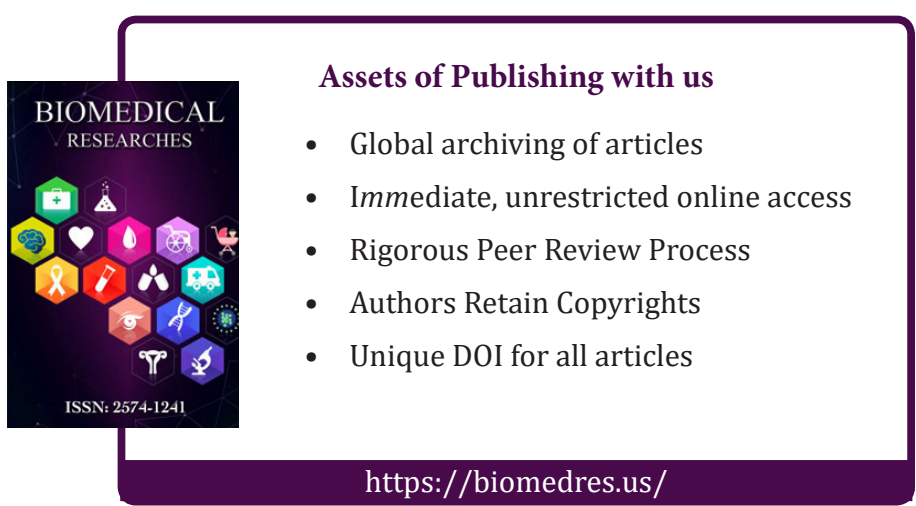

